\title{
CONTRIBUTION À L'ÉTUDE DES GROUPEMENTS RUPICOLES DES BOKKOYA (LITTORAL DU RIF CENTRAL, MAROC).
}

\author{
Ulrich DEIL \& Mohammed HAMMOUMI
}

\begin{abstract}
RESUME. Contribution à l'étude des groupements rupicoles des Bokkoya (Littoral du Rif Central, Maroc). Le massif calcaire des Bokkoya du littoral rifain est la partie la plus sèche des côtes méditerranéennes du Maroc. Après une introduction aux conditions physiques et phytochorologiques de la région, les groupements rupicoles sont décrits selon la méthode phytosociologique. Les falaises septentrionales sont couvertes par le Sedo wilczekiani-Sonchetum masguindalii ass. nov., une association endémique du sous-secteur NekorBokkoya (secteur Nekor-Triffa). Sur les pentes méridionales, on observe d'autres groupements appauvris du Poterion ancistroidis. Les fissures ombragées et nitrifiées au pied des falaises sont colonisées par le groupement à Mercurialis ambigua et Theligonum cynocrambe, les éboulis par celui à Succowia balearica. Sous des surplombs vivent des peuplements à Sarcocapnos enneaphylla. Les séries de contact sont illustrées par une figure. Un schéma syntaxonomique des groupements rupicoles du Nord du Maroc est présenté. Finalement, les associations et leurs vicariantes sont discutées dans le contex te ouest-méditerranéen.
\end{abstract}

Mots clé. Groupements rupicoles, Asplenietea, Maroc, Sonchus, phytochorologie

ABSTRACT. Rock communities in the Bokkoya Mountains (Coastal region of the Central Rif, Morocco). The coastal limestone massif of the Central Rif Mountains, the Bokkoya, is the driest part of the Moroccan mediterranean coast. After a brief introduction of its physical and phytochorological conditions, the rock communities are described in an phytosociological approach. The northern exposed cliffs are covered by the Sedo wilczekiani-Sonchetum masguindalii ass. nov., which is endemic in the subsector Nekor-Bokkoya of the phytochorological sector Nekor-Triffa. On southern slopes, we observe other fragmentary groups of the Poterion ancistroidis. Shady and nitrified rock fissures are colonized by the Mercurialis ambiguaTheligonum cynocrambe community, bolders by Succowia balearica. In halve caves there grow Sarcocapnos enneaphylla populations. The contact series are illustrated. A syntaxonomical scheme is given for the rock communities of Northern Morocco. Finally, the associations and their vicariants are discussed in the Westmediterranean context.

Key words. Rock communities, Asplenietea, Morocco, Sonchus, phytochorology

\section{INTRODUCTION}

La connaissance des groupements rupicoles du Maroc est encore assez limitée. Pour l'étage oromediterranéen du Haut Atlas et du Moyen Atlas oriental, on a des données assez précises (Quézel, 1952 et 1957). Pour les basses et moyennes altitudes du Moyen Atlas, les seules observations disponibles sont les études ponctuelles et fragmentaires de Braun- 
Blanquet \& Maire (1924). Récemment, Deil (1994a) a présenté quelques informations sur les Asplenietea du Tangérois et de la Dorsale Calcaire du Rif Occidental.

Les associations rupicoles du Rif Central ne sont pas encore étudiées en détail. Toutefois, dans le cadre d'une étude chorologique, phytosociologique et phytogéographique du versant mediterranéen du Rif Central, dont les résultats seront presentés ailleurs, nous avons pu réaliser quelques observations sur la végétation des milieux rocheux dans les basses altitudes qui sont presentées ici.

La discussion syntaxonomique des communautés végétales est basée sur une vue d'ensemble des groupements des Asplenietea trichomanis de la péninsule ibérique (Díaz Gonzalez 1989, Perez Carro et al. 1989), sur des études régionales dans d'autres parties de la chaîne bético-rifaine (Díez Garretas 1977, Martínez Parras \& Peinado Lorca 1990, Mota Poveda et al. 1991, Deil 1994a, Escudero \& Pajarón 1994) et pour le littoral oranais (Ouest de l'Algérie) sur les publications de Daumas et al. (1952) et Pons \& Quézel (1955).

\section{METHODES ET NOMENCLATURE}

Dans les années 1987 (Deil) et 1993 (Deil \& Hammoumi), 26 stations ont été étudiées. Les relevés et la classification des groupements ont été faits d'après la méthode phytosociologique (Braun-Blanquet 1964). La description des nouvelles associations suit les règles de Barkman et al. (1986). La nomenclature de Valdés et al. (1978) est adoptée pour les espèces végétales qu'on trouve aussi en Andalousie, celle de Jahandiez \& Maire (1931-1934) et Emberger \& Maire (1941) pour les endémiques marocaines (en partie modifiée par des révisions récentes).

La région dés éstudes. La figure 1 permet de préciser la localisation de la région des études, située sur le littoral rifain entre ElJebha et Al-Hoceima. Les Bokkoya forment une petite chaîne montagneuse, qui occupe la partie centrale de la région, delimitée à l'ouest par la vallée du Beni-Bou-Frah et la baie de Torres - Cala-Iris et à l'est par la ville de AlHoceima et l'embouchure des oueds Rhis et Nekor (fig. 2).

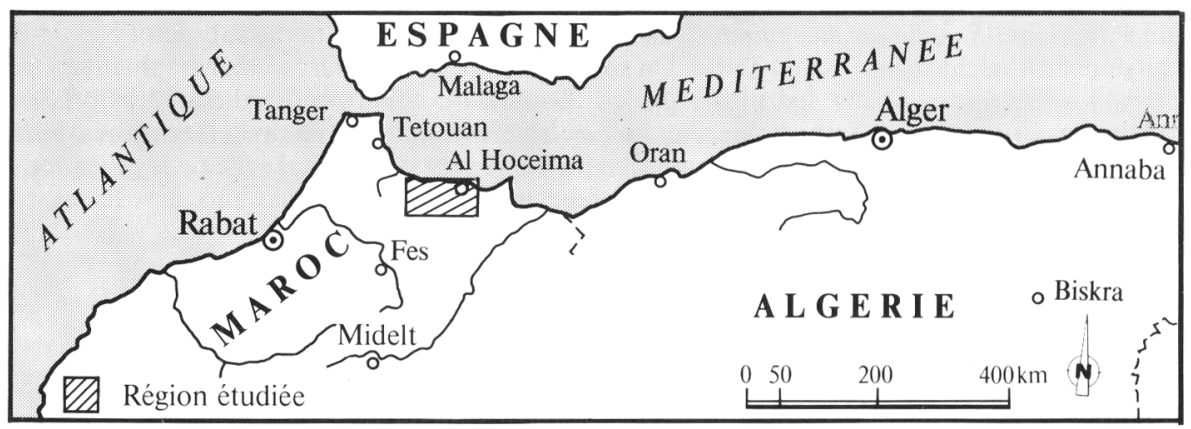

Figure 1: Plan de situation. Location of the study area. 
Le relief de la region est fortement accentué. Le massif des Bokkoya y apparaît comme un compartiment surélevé, culminant à $747 \mathrm{~m}$ d'altitude par rapport au reste du relief, dont il se sépare, respectivement à l'ouest et à l'est, par les vallées profondes des cours d'eau intermittents de l'oued Bades et de l'oued Tarmast. Sur son flanc méridional et interne sont individualisées une série de basses plaines internes le long de l'axe transverse allant de Snada par Rouadi jusqu'à Izemmouren. Depuis ces plaines on accède par un palier de reliefs collinaires, situés à une altitude moyenne de $500 \mathrm{~m}$, aux premiers versants septentrionaux de la chaîne du Haut Rif Central. Cette dernière se déploie en arc Nord - Nord-Ouest à Ouest Est de quelques 500 à $700 \mathrm{~m}$ de dénivellation par rapport aux bas reliefs, et les abrite du flux des perturbations atlantiques, ce qui explique leur aridité accentuée (pour les détails, voir la carte morphostructurale des Bokkoya dans Maurer 1968, fig. 113). Les falaises du littoral entre Bades et l'Ile de Topo sont presque inaccessibles.

La situation géologique est illustrée par la figure 3. Le massif des Bokkoya est dominé par les calcaires et dolomies du Trias et du Jurassique (nappe du jebel Bousicour) avec l'intercalation de quelques klippes paléozoïques (Andrieux, 1971; Carte Géologique du Rif 1/50000, Feuille Rouadi). Les bassins internes de l'époque SoltanoRharbien sont formés par des terrasses et plaines limoneuses, les pentes entre les Bokkoya et Targuist par le flysch de la nappe du Tizirène d'âge crétacé, les chaînes du Haut Rif Central par les schistes, les grès et les quartzites de la nappe de Ketama.

Le massif des Bokkoya est la partie la plus sèche du littoral rifain. La sécheresse accentuée est légèrement attenuée par les brumes et les brouillards fréquents et par l'humidité de l'air élevée du littoral.

Le climat y est typiquement

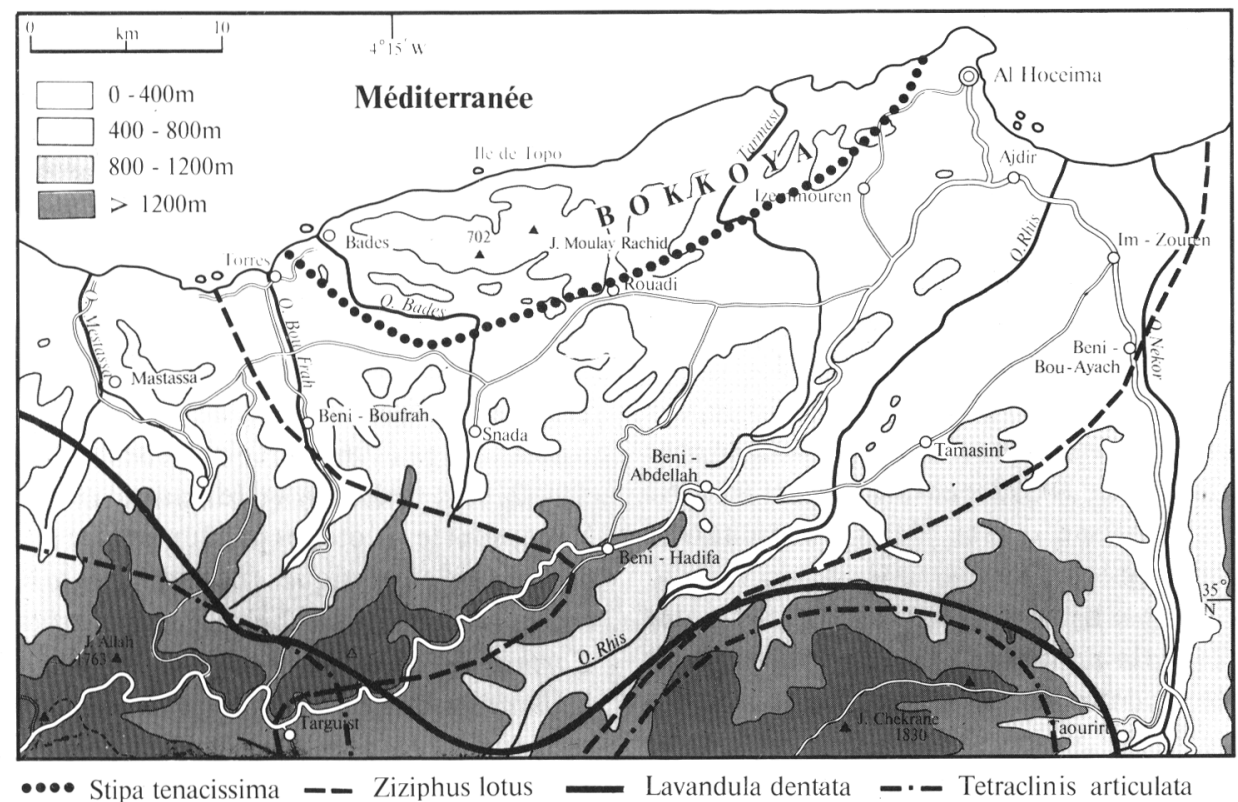

Figure 2: La région des études: relief et aire de distribution de quelques espèces climaciques. The study area: relief features and distribution of some climax plant species. 


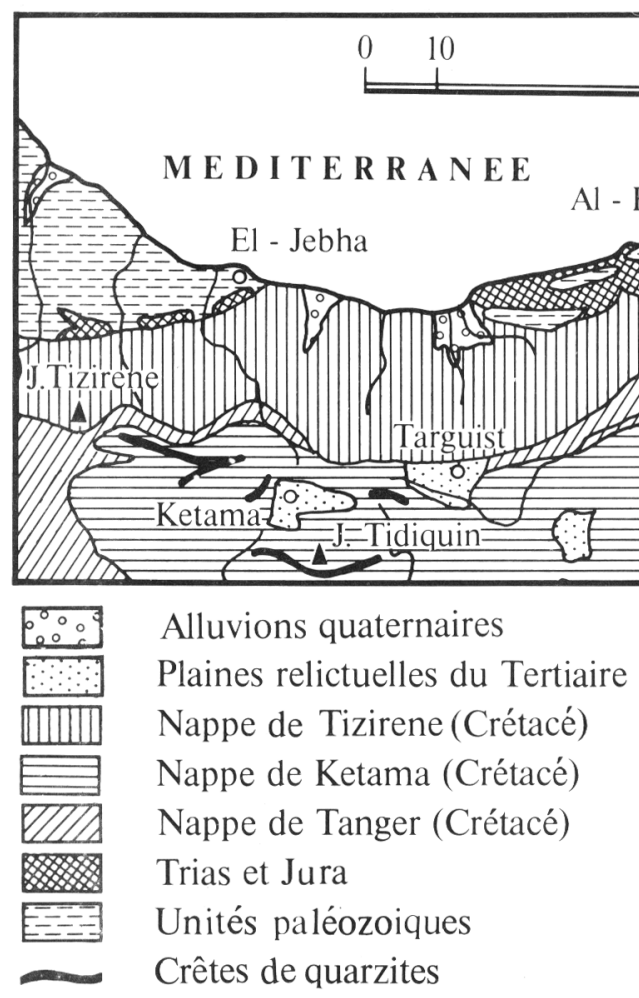

- Figure 3: Carte géologique. Geological map.

méditerranéen. Le maximum des précipitations tombe pendant les mois de novembre à mars/ avril (voir le climagramme de El-Jebha, fig. 4). Les précipitations annuelles diminuent de l'ouest vers l'est (de $330 \mathrm{~mm}$ à El-Jebha vers $300 \mathrm{~mm}$ à Al-Hoceima), de façon plus accentuée sur la côte rifaine que sur la côte bétique. La climatogenèse de cette différenciation pluviométrique est discutée en détail par Couvreur-Laraichi (1972). Elle souligne une homologie climatique du secteur bokkoyense avec la Sierra del Gador entre Motril et Almeria qui s'exprime aussi dans la situation biogéographique.

La position phytogéographique des Bokkoya, la sectorisation de la chaîne rifaine et l'étude des étages bioclimatiques seront les thèmes d'une autre publication. Dans le cadre de cette étude, deux aspects doivent être soulignés:

1. La pénetration des éléments
Figure 4: Climagramme d'une station côtière. Climagraph of a coastal station.

thermoméditerranéens (comme Rhus pentaphylla et Periploca laevigata) et même steppiques (comme Stipa tenacissima et Psychine stylosa) dans cette partie côtière du Rif justifie la division phytogéographique du Nord du Maroc proposée par Sauvage \& Vindt (1952) plutôt que celle de Rivas-Martínez (1981).

La figure 2 montre la distribution régionale de quelques espèces indicatrices des étages thermo- et inframéditerranéens. Le massif des Bokkoya est la seule région du Mahgreb où on trouve des grands peuplements de Stipa tenacissima dans une situation littorale. Les limites d'aires de Tetraclinis articulata et Lavandula dentata correspondent exactement avec la limite chorologique entre le secteur Rif-Nord Est et le secteur NekorTriffa sensu Sauvage \& Vindt (1952). La carte 1 de Sauvage (1961) précise cette limite.

2. Les Bokkoya et le bassin versant du Nekor sont les écotopes pour un certain nombre d'espèces endémiques comme Perralderia (=Fontquera) paui, Sonchus masguindalii, Carthamus riphaeus, Teucrium gypsophilum, T. huotii var. grosii, Misopates chrysothales etc. (Jahandiez \& Maire 1931-1934). Par ailleurs, d'autres taxons endémiques comme Sideritis fontiqueriana ont été découverts par des études taxonomiques approfondies (Peris 
et al. 1995). La dernière était traitẹe comme une sous-espèce de $S$. subatlantica jusqu'à récemment. Ces espèces endémiques permettent de caractériser le sous-secteur Nekor-Bokkoya du secteur Nekor-Triffa. Ce sous-secteur est homologue du point de vue bioclimatique (Couvreur-Laraichi 1972) et biogéographique au sous-secteur Gadorense sensu Rivas-Martínez et al. (1991) en Espagne. Des éléments floristiques communs de ces deux côtés de la mer d'Alboran sont par exemple Lapiedra martinezii et Galium ephedroides. Le sous-secteur Nekor-Bokkoya est aussi caractérisé par des irradiations d'espèces oranaises-est-rifaines comme Chiliadenus (=Jasione) rupestris et Galium brunnaeum et moulouyienne-bokkoyienne comme Centaurea involucrata (Vogt \& Oberprieler in press), qui arrivent jusqu'à Bades.

La région d'étude se caractérise par une diversité biologique remarquable qui se traduit tant au niveau floristique qu'au niveau de la faune terrestre et marine. Les falaises littorales des Bokkoya sont colonisées par la population la plus importante du Balbuzard pêcheur (Pandion haliaetus) en Mediterrannée et par l'unique colonie du Goéland d'Audouin (Larus audouinii) au Maroc. Avella \& Gonzalez (1984) ont souligné la nécessité d'établir des réserves biologiques pour stabiliser la population menacée des phoques (Monachus monachus) du littoral méditerranéen du Maroc. Une colonie permanente de cette espèce est supposée dans la région des Bokkoya. Pour sauver la richesse biotique de la région, on a envisagé la création d'un Parc National d'Al-Hoceima (Ribi, 1992).

\section{RESULTATS}

Les groupements rupicoles. Pour mieux voir la valeur sociologique et le comportement écologique des espèces des milieux rupicoles des Bokkoya, la totalité des 26 relevés est presentée dans un seul tableau (tab. 1). Ils sont arrangés selon un gradient d'humidité et de nitrophilie: le tableau commence par un groupement colonisant les falaises septentrionales du littoral (colonne 1-15), qui passe à des groupements des falaises à l'intérieur du massif (col. 16-17 et 18-19), suivis par des groupements nitrophiles, situés sur des éboulis au pied des falaises (col. 20) ou des fissures ombragées dans les bas fonds des falaises (col. 21-23). Le tableau se termine par un groupement sciaphile des balmes ( $=$ grotte ouverte au-dessous des surplombs)(col. 2426). La localisation des sites des relevés est donnée en fig. 5.

\section{Sedo wilczekiani-Sonchetum masguindalii} ass. nov.

Le Sedo-Sonchetum (tab. 1, colonne 115) est un groupement permanent des falaises verticales littorales en exposition nord et nordest. Les stations sont souvent arrosées par des brouillards. La surface des rochers est couverte par des microstalactites et des lichens du genre Roccella. Les peuplements phanérogamiques sont ouverts (recouvrement total entre 5 et $25 \%)$. Les plantes sont enracinées dans des fissures de rochers. L'association est bien caractérisée pas les deux taxons, qui ont été choisi pour nommer cette phytocénose.

Sonchus masguindalii Pau \& Font-Quer est endémique dúlittoral rifain entre Bades et l'embouchure du Nekor. La distribution principale de Sedum hirsutum All. subsp. wilczekianum (F.-Q.) Maire se trouve dans les Bokkoya; des populations isolées se rencontrent sur les rochers à Mouláy-Idris et au jebel Zerhoun.

À part la sous-association typicum (col. 4-12, rel. type 9), nous avons pu observer deux autres sous-types. La sous-associatior silenetosum obtusifoliae subass. nov. (col. 13 , rel. type 2) se trouve entre 5 et $30 \mathrm{~m}$ d'altitude (figs. 5 et 6), où le milieu est influencé par les embruns salés. Cette sous-association est différenciée du type par un nombre élevé 


\begin{tabular}{|c|c|c|c|c|c|c|c|c|c|c|c|c|c|c|c|c|c|c|c|c|c|c|c|c|c|c|}
\hline \multirow[t]{2}{*}{ Colonne } & & & & & & & & & & 1 & 1 & 1 & 1 & 1 & 1 & 1 & 1 & 1 & 1 & 2 & 2 & 2 & 2 & 2 & 2 & 2 \\
\hline & 1 & 2 & 3 & 4 & 5 & 6 & 7 & 8 & 9 & 0 & 1 & 2 & 3 & 4 & 5 & 6 & 7 & 8 & 9 & 0 & 1 & 2 & 3 & 4 & 5 & 6 \\
\hline \multirow[t]{2}{*}{ Altitude $(x 10 \mathrm{~m})$} & & & & & & & & 2 & 2 & 2 & 1 & 1 & 4 & 1 & 1 & 7 & 5 & 5 & 4 & 7 & 1 & 5 & 2 & 4 & 1 & 1 \\
\hline & 1 & 3 & 1 & 1 & 1 & 2 & 1 & 0 & 0 & 0 & 0 & 0 & 5 & 5 & 2 & 0 & 0 & 0 & 5 & 0 & 5 & 0 & 0 & 5 & 0 & 0 \\
\hline \multirow[t]{2}{*}{ Inclinaison ( $\mathrm{i}=$ inverse) } & 3 & 8 & 8 & 8 & 9 & 8 & 8 & 8 & 8 & 9 & 9 & 9 & 9 & 9 & 9 & 8 & 8 & 9 & 8 & 2 & & 2 & 5 & & & \\
\hline & 0 & 0 & 0 & 0 & 5 & 5 & 0 & 5 & 0 & 0 & 0 & 0 & 0 & 0 & 0 & 5 & 0 & 0 & 0 & 0 & 0 & 0 & 0 & $\mathrm{i}$ & $\mathrm{i}$ & i \\
\hline \multirow[t]{2}{*}{ Exposition } & & $\mathrm{N}$ & $\mathrm{N}$ & & & $\mathrm{N}$ & $\mathrm{N}$ & & $\mathrm{N}$ & $\mathrm{N}$ & $\mathrm{N}$ & $\mathrm{N}$ & $\mathrm{N}$ & $\mathrm{N}$ & $\mathrm{N}$ & $S$ & & $N$ & & S & & $\mathrm{N}$ & $\mathrm{N}$ & $\mathrm{N}$ & & $\mathrm{N}$ \\
\hline & $\mathrm{N}$ & $\mathrm{O}$ & $\mathrm{O}$ & & $\mathrm{N}$ & $\mathrm{O}$ & $\mathrm{O}$ & $\mathrm{N}$ & $\mathrm{O}$ & $\mathrm{O}$ & $\mathrm{OC}$ & $\mathrm{O}$ & & $\mathrm{E}$ & $\mathrm{O}$ & & $\mathrm{O}$ & $\mathrm{E}$ & S & $\mathrm{E}$ & . & $\mathrm{O}$ & $\mathrm{O}$ & $\mathrm{O}$ & $\mathrm{N}$ & $\mathrm{O}$ \\
\hline \multirow[t]{2}{*}{ Recouvrement total en $\%$} & 7 & 2 & 3 & & & 1 & 2 & 2 & 2 & 1 & 1 & 2 & 2 & 2 & 1 & 2 & 1 & 2 & 2 & 3 & 6 & 2 & 2 & 1 & & 1 \\
\hline & 0 & 0 & 0 & 5 & 5 & 0 & 5 & 0 & 0 & 0 & 5 & 0 & 0 & 0 & 0 & 0 & 0 & 0 & 0 & 0 & 0 & 0 & 0 & 0 & 5 & 0 \\
\hline
\end{tabular}

ECAS Sedo wilczekiani-Sonchetum masguindalii

Sonchus masguindalii

Sedum hirsutum wilczekianum

EDI stipetosum tenacissimae

Stipa tenacissima

Lapiedra martinezii

Anthyllis podocephala

Bupleurum gibraltaricum

ECAL Poterion ancistroidis

Chiliadenus rupestris

Putoria brevifolia

Galium ephedroides

Galium brunnaeum

ECSO Tinguarrenalia siculae

Sedum gypsicola glandulosum

Lavatera maritima

Calendula suffruticosa s.str.

Fumaria rupestris

Ferula tingitana

Athamantha sicula

Polygala rupestris

Cheilanthes vellea

ECOR Asplenietalia petrarchae

Piptatherum caerulescens

Melica minuta

Cheilanthes acrosticha

Antirrhinum barrelieri

Ficus carica (plantule)

Phagnalon rupestre

ECCL Asplenietea trichomanis

Sedum sediforme

Phagnalon saxatile

Saxifraga globulifera

Asplenium ceterach

E transgressives des Crithmo-Limonietea

Asteriscus maritimus

Daucus carota hispidus

Crithmum maritimum

Limonium cossonianum-groupe

Silene obtusifolia

$+13122112212222$

Centaurca fragilis

Limonium emarginatum

. . $1+.1+1 .+++$

Succowia balearica

Ballota hirsuta

Psoralea bituminosa

ECAS à Mercurialis ambigua et Theligonum cynocrambe

Mercurialis annua ambigua

Theligonum cynocrambe

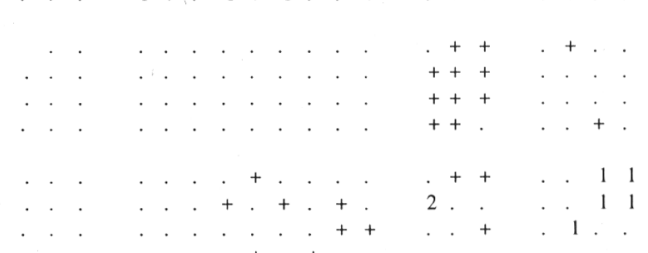

ES des Parietarietalia et Geranio-Cardaminetalia

Geranium molle

Geranium purpureum

Valantia muralis

Campanula erinus

Valantia hispida

EComp ruderales

Fedia cornucopiae

Sherardia arvensis
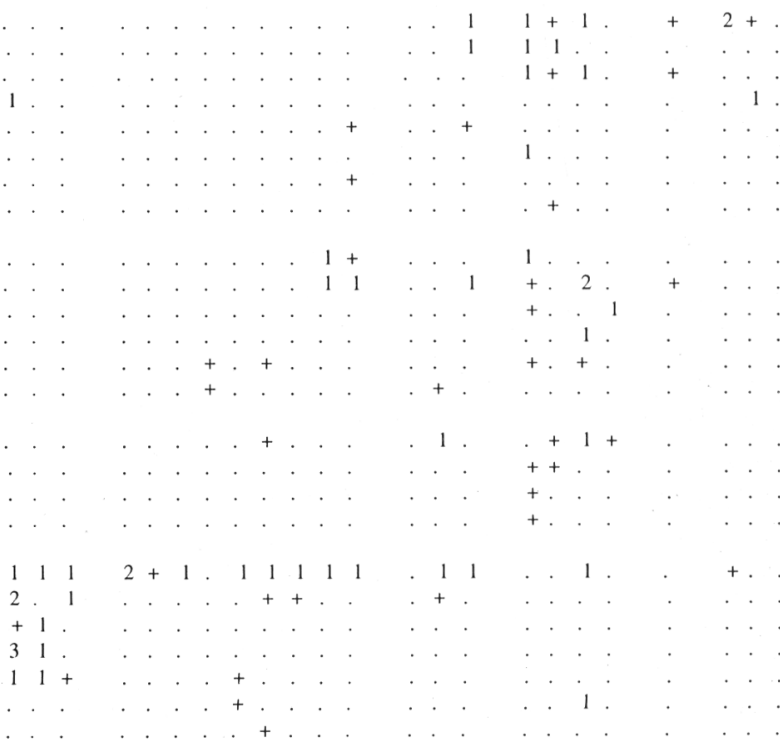

$\begin{array}{lll}1 & 2 & 1\end{array}$

222 


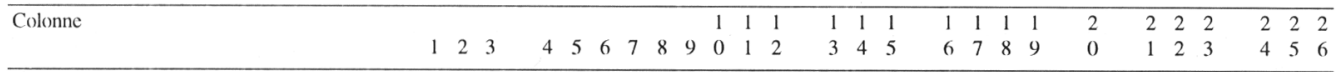

EC du Sarcocapnetum enneaphyllae

Rosmarmo-Ericion, Ulici-Rosmaricion tournefortii, Ononido-Rosmarin. Lavandula dentata

Micromeria inodora

Launaca arborescens (plantule)

Viola arborescens

Ornithogalum sessiliflorum

Cytisus purgans balansae

Erica multiflora

Genista tricuspidata duriaei

Thymus serpylloides gadorensis

Periplocion, Tetraclini-Pistacenion, Pistacio-Rhamnetalia

Ephedra fragilis s.str.

Periploca laevigata angustifolium

Withania frutescens

Pachycymbium decaisneanum

Chamaerops humilis

Tetraclinis articulata

Prasium majus

Olea curopaea sylvestris

Arisarum simorrhinum

Osyris quadripartita

Pistacia lentiscus

Lycium intricitum

Autres espèces

Telephium imperati

Aristolochia pistolochia

Dactylis glomerata hispanica

Brachypodium distachyon

Urginea maritima

Maucanthemum decipiens

Allium subvillosum

Sonchus tenerrimus

Silene divaricata

Anthericum baeticum
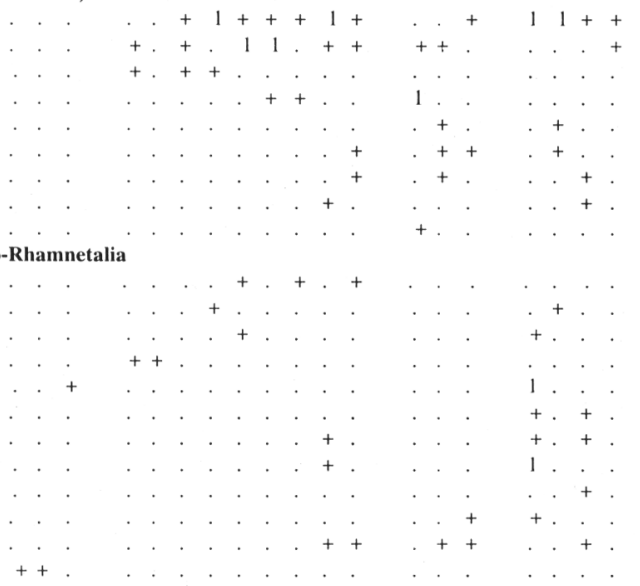

$+$
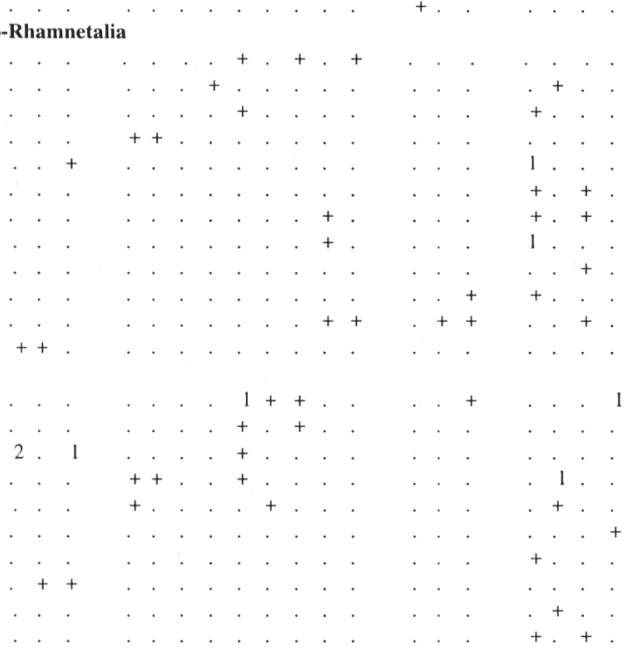

Espèces rares: rel. 2: Mesembryanthemum nodiflorum +; Desmazeria marina + ; Frankenia corymbosa +; rel. 3 : Beta vulgaris maritima r; rel. 4 : Hyparrhenia hirta 1 ; rel. 5 : Asphodelus ramosus + ; Lagurus ovatus + ; rel. 6: Daphne gnidium + ; rel. 8: Coronilla valentina glauca + ; rel. 13 : Ampelodesmos mauritanica 1; Teucrium fruticans + ; Cistus albidus +; rel. 14: Rosmarinus officinalis 1; rel. 16 : Calicotome intermedia + ; Ruscus hypophyllum + ; rel. 17 : Linaria spec. + ; Lobularia maritima 1 ; Fumana thymifolia + ; Sedum rubens + ; Lavandula multifida 1 ; Pancratium maritimum + ; rel. 18 : Lithodora fruticosa + ; Carex hallerana + ; rel. 21 : Trigonella monspeliaca + ; Bromus rubens 1 ; Hedypnois cretica + ; Filago lutescens + ; Fumana ericoides + ; rel. 22 : Stellaria media + Campanula dichotoma kremeri + ; Picris echioides + ; Euphorbia esula + ; Umbilicus rupestris + ; rel. 23 : Torilis nodosa +; Anagallis arvensis +; Sideritis romana +; Centranthus calcitrapae + ; Galium murale +; Ononis hispanica ramosissima 1 .

$\mathrm{EC}=$ espèces caracteristiques; $\mathrm{ECAS}=$ espèces caracteristiques de l'association; $\mathrm{ECAL}=$ espèces caracteristiques de l'alliance; ECOR = espèces caracteristiques de l'ordre; ECSO = espèces caracteristiques de le subordre; ECCL = espèces caracteristiques de la classe; EDI = espèces differentielles; ET = espèces transgressives; EComp = espèces compagnard

Table 1: Groupements rupicoles des Bokkoya. Rock communities of the Bokkoya Mountains. 


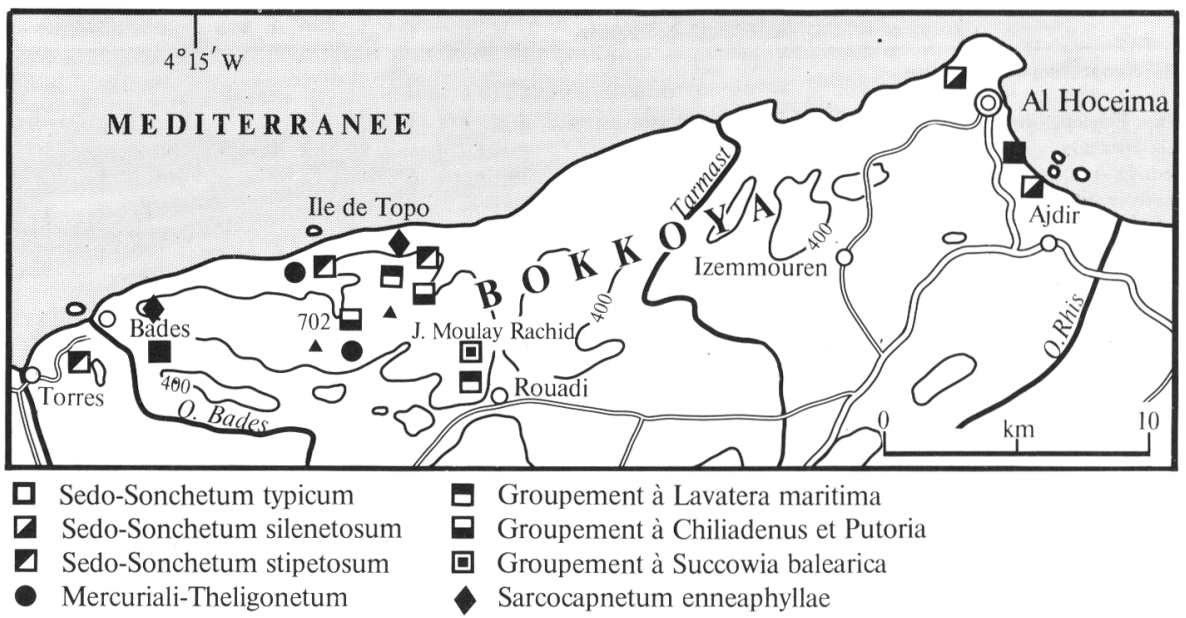

Figure 5: Localisation des relevés des groupements rocheux. Sampling localities of the rock communities.

d'espèces sténohalines du Dauco-Asteriscetum maritimi Wojterski 1988 (Asteriscion maritimi Gehu et al. 1992, Crithmo-Limonietea) comme Silene obtusifolia et Crithmum maritimum. Dans le Rif semi-aride, Asteriscus maritimus n'est pas strictement liée au littoral. Elle accompagne toutes les stations du SedoSonchetum. La différence entre l'Asteriscion maritimi Géhu et al. 1992 et le Daucion halophili Rivas-Martínez et al. 1990 reste à préciser. La sous-association stipetosum tenacissimae subass. nov. (col. 13-15, rel. type 15) a été observée dans les parties centrales des Bokkoya (voir la distribution de Stipa tenacissima, fig. 2). C'est un groupement transitoire vers les groupements steppiques du Stipo tenacissimae-Launeion acanthocladae Djebaili 1990 respectivement le LapiedroStipetum tenacissimae Rivas-Martínez \& Alcaraz in Alcaraz 1984 s.l. avec des espèces differentielles comme Stipa tenacissima, Lapiedra martinezii et Anthyllis podocephala.

Synsystématique: La délimitation des alliances de la classe Asplenietea trichomanis reste à clarifier par un plus grand nombre de données. Pour le moment, cette association nouvelle n'est pas à intégrer dans les alliances des Asplenietalia petrarchae, qui ont été décrites de l'arc bético-rifain (Cosentinio bivalentis-Lafuention rotundifoliae Asensi et al. 1990, Campanulion velutinae Martínez Parras et al. 1990 et Teucrion buxifolii Rivas Goday 1955). On trouve un certain nombre de taxons des ordres Asplenietalia petrarchae Br.B1. \& Meier 1934 et Tinguarretalia siculae Daumas et al. 1952. Malheureusement, Escudero \& Pajaron (1994) n'ont pas inclus les données nord-africaines dans leur analyse numérique des Asplenietalia petrarchae. Pour cela on adopte ici la position de Galán de Mera (in Pérez Latorre et al. 1996), qui traite les Tinguarretalia siculae sensu Daumas et al. (1952) comme sous-ordre des Asplenietalia petrarchae.

Dans la région des Bokkoya, on est juste à la limite phytochorologique des espèces bético-rifaines comme Antirrhinum barrelieri et Bupleurum gibraltaricum et celles de distribution rifaine-oranaise comme Chiliadenus rupestris et Galium brunnaeum. 
Daumas et al. (1952) ont proposé pour les groupements rupicoles de l'Oranais l'alliance Rupicapnion africanae Daumas et al. 1952. D'après nos observations au jebel Musa (Deil, 1994a) et dans le Moyen Atlas (Deil, ined.) et d'après les données de Perez Latorre et al. (1995) des montagnes sudibériques, Rupicapnos africana śubsp. decipiens est sciaphile comme les espèces du genre Sarcocapnos. Quand on prend comme association basale du Rupicapnion l' «Ass. à Fumaria (Rupicapnos) africana Br.-Bl. \& Maire 1924» (incluant le Rupicapnetum africanae sensu Perez Latorre et al. 1995), les associations du Rupicapnion sont définies comme groupements des balmes des chaînes bétique, du Maroc Oriental et de l'Algérie Occidentale.

Pour cette raison, nous proposons pour les associations des rochers, directement exposées à la lumière, de les inclure dans l'alliance du Poterion ancistroidis Br.-Bl. in Meier \& Br.-Bl. 1934 em. Deil \& Galán de Mera in press. Elle s'étend du Bokkoya jusqu'à Oran et y est caractérisée par Chiliadenus (= Jasonia) rupestris, Galium brunnaeum, $G$. ephedroides et Putoria brevifolia. Elle est incluse dans l'ordre des Asplenietalia petrarchae Br.-Bl. \& Meier in Meier \& Br.-Bl. 1934, dans le sous-ordre des Tinguarrenalia siculae (Daumas et al. 1952) Galán de Mera in Perez Latorre et al. 1996 et dans la classe des Asplenietea trichomanis (Br.-Bl. in Meier \& Br.-B1.) Oberd. 1977 (voir le schéma syntaxonomique à la fin du texte). Une vue d'ensemble des groupements rupicoles calcaires du Maroc est donnée par Deil \& Galán de Mera (in press).

2. Groupements appauvis du Poterion ancistroidis

Sedum hirsutum et Sonchus masguindalii restent limités aux falaises du littoral et à celles exposées au nord. A l'intérieur des Bokkoya et en exposition sud, les sites rocheux sont rares et notre connaissance de leur végétation est encore fragmentaire. Quatre relevés ont été réalisés ici (fig. 5): Deux (col. 16 et 17) avec Lavatera maritima, une Malvacée arbustive de distribution ouest-méditerranéene, toujours liée aux rochers calcaires maritimes; deux autres relevés (col. 18 et 19) avec Putoria brevifolia, une Rubiacée rupicole de distribution ibéro-mauritanienne. Dans l'Oranais, Putoria caractérise le Campanulo oranensis-Putorietum Daumas et al. 1952. Dans le Rif, nos observations sont trop préliminaires pour définir ces groupements sous forme d'associations.

\section{Groupement à Succowia balearica}

Succowia balearica est une Brassicacée exozoochore de distribution ouestméditerranéenne. Elle est toujours liée au milieu nitrophile du pied des falaises, où les troupeaux passent le midi à l'ombre. Aux environs d'Oran, Daumas et al. (1952) ont observé cette espèce associée à Corydalis heterocarpa, Acanthus mollis et Smyrnium olusatrum. Une telle combinaison floristique à été constatée aussi à Gibraltar (Deil 1994a). Le Succowio-Ceratocapnetum heterocarpae Daumas et al. 1952, bético-oranais, est vicariant du Succowio-Smyrnietum Bartolo et al. 1990 de Sicile. Dans les Bokkoya, la combinaison des espèces (tab. 1, col. 20) ressemble plus à un peuplement à Succowia observé par Cantó et al. (1986) au pied des falaises du Peñon de Ifach à Calpe (Espagne). Les groupements à Succowia restent à étudier en détail. Ils font partie du Geranio-Anthriscion (Geranio-Cardaminetalia hirsutae Brullo 1985) et du Allion triquetri (UrticoScrophularietalia peregrinae Brullo 1985).

4. Groupement à Mercurialis ambigua et Theligonum cynocrambe

Theligonum cynocrambe est de distribution circum-méditerranéenne. Cette 
Theligonacée à dispersion myrmecochore habite toujours des fissures de rochers et des murailles nitrifiées et ombragées. La surface des rochers est souvent couverte par des lichens ornithocoprophiles. Le groupement à Mercurialis ambigua et Theligonum cynocrambe (tab. 1, col. 21-23) à été observé dans des éboulis calcaires des Bokkoya à l'Ile de Topo et au pied d'une falaise du jebel Moulay Rachid (fig. 5) ainsi que dans la région du Détroit de Gibraltar (à Ksar Srhir et à Beni Younech)(Deil 1994a). A part les taxons choisis pour le nommer, il est caractérisé par les espèces du Valantio-Galion muralis et du Geranio-Anthriscion (GeranioCardaminetalia hirsutae Brullo 1985). Ce groupement est vicariant du Mercuriali ellipticae-Theligonetum cynocrambes (Fndz. Casas 1972) Peinado et al. 1986 de la Sierra del Pinar (Andalousie) et du Geranio pusilliTheligonetum cynocrambes Rivas-Martínez 1978 de l'Algarve (Portugal).

\section{Groupement à Sarcocapnos enneaphylla}

La Fumarioidée Sarcocapnos enneaphylla, largement distribuée dans les montagnes ibériques, a ses seules stations maurétaniennes dans la région des Bokkoya (Liden 1986). Elle habite des grottes à l'eau suintante épisodique. Les plantes sont abritées des précipitations directes par les balmes, elles ne reçoivent que des radiations indirectes. Dans les Bokkoya, nous avons observé des stations au jebel Moulay-Rachid et sur les versants de l'oued Bades (tab. 1, col. 24-26, fig. 5).

Quand les critères d'homogénité sont appliqués d'une manière stricte (comme ici) et la surface des relevés est restreinte au milieu des balmes, les peuplements à Sarcocapnos sont presque monospécifiques. Une hiérarchisation d'un tel groupement par l'approche classique de Braun-Blanquet (1964) reste douteuse, l' approche des coeno-syntaxons (voir dernier chapitre) semble plus appropriée. On peut interpréter le Sarcocapnetum enneaphyllae comme une association basale de l'ordre des Sarcocapnetalia enneaphyllae Fernández Casas 1972.

La plupart des auteurs ont choisi des surfaces hétérogènes, leurs relevés contiennent aussi des espèces caractéristiques des Parietarietalia, des Asplenietalia petrarchae ou des Potentilletalia; raison pour laquelle aussi Díaz González et al. (1989) ont proposé d'inclure les différents sous-types du Sarcocapnetum dans ces différents syntaxons.

Toposequences de la végétation des falaises. Quelques séries de contact entre le Sedo-Sonchetum et d'autres associations sont illustrées par la figure 6. Dans des situations salines, il est remplacé vers le bas versant de la falaise par le Dauco-Asteriscetum, le Limonietum emarginati ou des groupements des Frankenietea, dans des situations nitrifiées par le groupement à Succowia balearica ou celui à Mercurialis ambigua et Theligonum cynocrambe. Colonisant un surplomb, il est en contact avec le Sarcocapnetum enneaphyllae. Une toposéquence vicariante est constatée par Loidi \& Galán de Mera (1989) dans la Sierra de Guadarrama (Cheilantho acrostichaeAsplenietum petrarchae jasonietosum glutinosae et Chaenorhino segoviensisSarcocapnetum enneaphyllae).

Vers le haut des falaises, le SedoSonchetum est suivi par le Lapiedro-Stipetum Riv.-Mtz. \& Alcaraz, 1984. Cet enchaînement ressemble à la série caténale de Gibraltar avec le Biscutello-Iberidetum et un groupement à Stipa tenacissima et Rosmarinus officinalis (Deil 1994a). Si les versants sont moins inclinés et les sols plus évolués, le Sedo-Sonchetum et les autres groupements du Poterion ancistroidis sont en contact avec des associations préforestières comme le Calicotomo intermediae-Tetraclinetum articulatae Barbero et al. 1981, le Periploco angustifoliaeTetraclinetum articulatae Benabid 1984 ou avec des groupements de substitution comme 


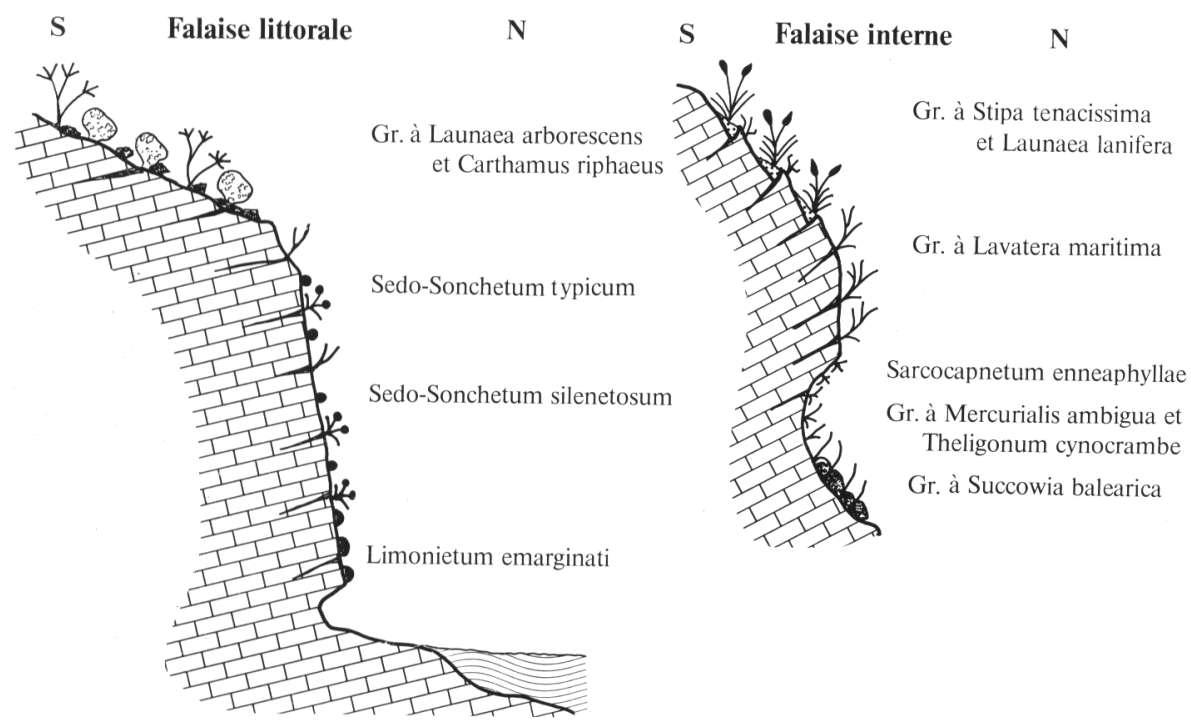

S Falaise côtière

N

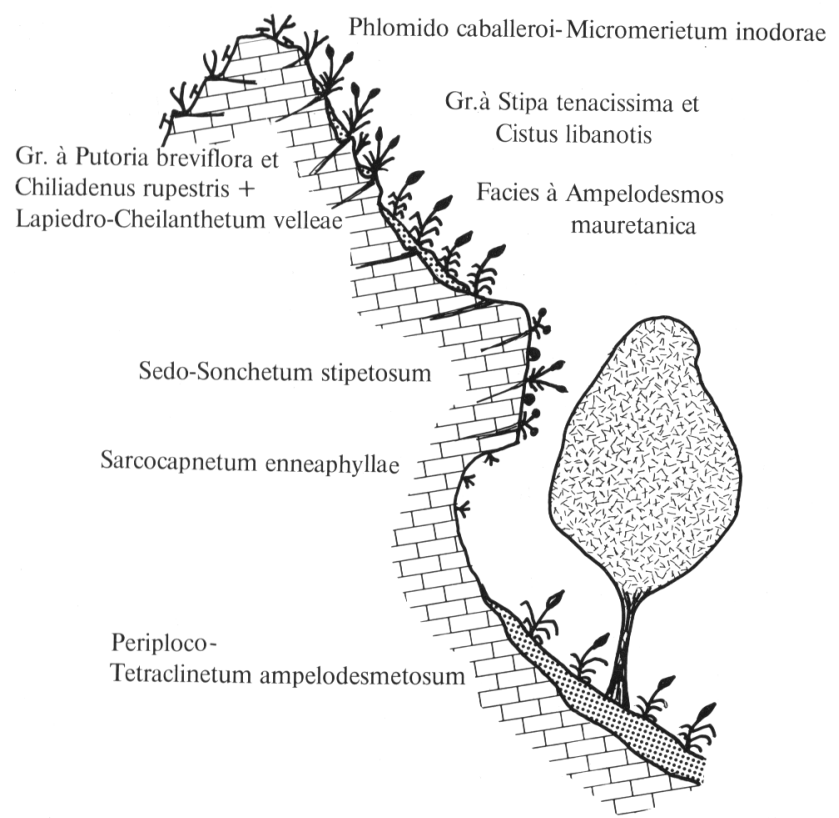

Figure 6: Toposéquences de la végétation des falaises. Contact series of plant communities on cliffs. 
le Phlomido caballeroi-Micromerietum inodorae Quézel et al. 1988 et une association à Launaea arborescens et Carthamus riphaeus (Deil, ined.).

Pour compléter les complexes végétaux de falaises, il serait nécessaire d'inclure les associations épilithiques de lichens. Mais pour cette catégorie systématique, on est encore au niveau de la recherche floristique. Alonso \& Egea (1994) ont découvert sur les falaises calcaires aux environs de Al-Hoceima toute une série de lichens, nouveaux pour le Maroc.

Espèces et groupements vicariants. Sonchus masguindalii Pau \& Font-Quer est synonyme de Sonchus tenerrimus L. ssp. pustulatus (Willk.) Batt. var. masguindalii (Pau \& F.-Q.) Maire sensu Jahandiez \& Maire (1934). Boulos (1973) a rétabli le rang spécifique de ce taxon. Cette plante chamaephytique avec un feuillage nettement succulent appartient à la section Pustulatus. Ce groupe de Sonchus, avec ses caractères primitifs et relictuels, a évolué directement à partir du sous-genre Dendrosonchus.

Les taxons de la section Pustulatus forment une mosaïque de taxons vicariants dans des groupements des rochers calcaires autour de la mer d'Alboran (Boulos 1972, 1973): Sonchus pustulatus Willk. (dispersé de Almeria, le long de la Costa del Sol jusqu'à Tanger et à Oran) est associé aux CrithmoLimonietea (Blanca López \& Cueto Romero 1985), Sonchus fragilis Ball. (distribution hosmarienne) caractérise le Soncho fragilisRhodanthemetum laouense (Deil 1994a), Sonchus masguindalii le Sedo-Sonchetum (hic loco)(bokkoyenne) et Sonchus briquetianus Gandoger colonise les rochers des Iles Jaffarines dans l'embouchure de la Moulouya.

Le phénomène de l'évolution de taxons vicariants dans les groupements rupicoles ouest-méditerranéens et en particulier béticomauritaniens peut être constaté parmi d'autres taxons comme Biscutella, Rhodanthemum (Vogt 1991, 1994), Campanula de la section Mediae (Quézel 1953), les Silénes du groupe S. rosulata et $S$. mollissima (Jeanmonod \& Bocquet 1981, Jeanmonod 1984, 1985), les Fumarioideae (Rupicapnos, Sarcocapnos, Fumaria)(Liden 1986) et Limonium. Pour quelques-uns (Silene, Fumarioideae), les chercheurs ont esquissé des tendances évolutives très claires et ont formulé une hypothèse sur les routes migratoires pendant l'ère Tertiaire. Le comportement sociologique des taxons laisse supposer une coévolution dans le complexe caténal des falaises (pour les détails voir Deil 1994a).

Pons \& Quézel (1955) ont discuté, sur l'exemple du genre Limonium dans les groupements rupicoles du Oranais, les conséquences d'une pulvérisation taxonomique pour la syntaxonomie. Ils ont formulé leur sentiment de malaise de définir un grand nombre d'associations par des taxons vicariants très localisés par la remarque suivante: «En conclusion, un essai de rationalisation des groupements des rochers maritimes sur une grande longueur de côte conduit à mettre en évidence le caractère subordonné des espèces rares et localisées et, d'autre part, à montrer la nécessité d'une certaine liberté dans les méthodes de hiérarchisation phytosociologique, de façon à tenir compte de caractères propres - richesse floristique, caractères écologiques - de la végétation étudiée.»

Une solution pour la hiérarchisation des associations riches en espèces endémiques et relictuelles est l'approche phytosociologique de Schuhwerk (1990), qui utilise les termes «forme historique normale» et «forme historique relictuelle». Une autre possibilité est le concept des coeno-syntaxons (Deil 1992, 1994a, 1994b), permettañt la caractérisation des syntaxons par des taxons supraspécifiques. 
Schema syntaxonomique des groupements rupicoles du Nord du Maroc. Le schéma suivant donne une vue d'ensemble des groupements rupicoles de l'arc rifain, qui ont été décrits par Daumas et al. (1952), pal Deil (1994a), dans cette contribution et dans une synthèse sur les Adiantetea du Maroc (Deil, 1996).

ASPLENIETEA TRICHOMANIS (Br.-Bl. in Meier \& Br.-Bl. 1934) Oberdorfer 1977

Asplenietalia petrarchae Br.-Bl. \& Meier in Meier \& Br.-Bl. 1934

Tinguarrenalia siculae (Daumas et al. 1952) Galán de Mera in Perez Latorre et al. 1996

Poterion ancistroidis Br.-Bl. in Meier \& Br.-Bl- 1934 em. Deil \& Galán de Mera in press

Sedo wilczekiani-Sonchetum masguindalii ass. nov.

sonchetosum

silenetosum obtusifoliae subass. nov. stipetosum tenacissimae subass. nov. Campanulo tlemcensis-Teucrietum buxifolii Daumas et al. 1952

Rupicapnion africanae Daumas et al. 1952

Ass. à Fumaria (Rupicapnos) áfricana Br.-Bl. \& Maire 1924

(incl. Groupement à Rupicapnos decipiens Deil 1994 et Rupicapnetum africanae Perez Latorre et al. 1995)

Campanulion velutinae Martínez Parras et al. 1990

Soncho fragilis-Rhodanthemetum laouense Deil 1994

Stachyo circinatae-Rhodanthemetum hosmariense Deil 1994

Groupement à Campanula velutina et Parietaria judaica Deil 1994

Coeno-Sarcocapnetalia Deil \& Galán de Mera in press
Coeno-Sarcocapnion Deil \& Galán de

Mera in press

Sarcocapnetum enneaphyllae Rivas Goday 1941

Sarcocapno crassifoliae-Erodietum tordylioidis Daumas et al. 1952

Anomodonto-Polypodietalia O. Bolós \& J. Vives in O. Bolós 1957

Bartramio-Polypodion cambrici $O$. Bolós \& J. Vives in O. Bolós 1957

Davallio canariensis-Sedetum (hirsuti) baetici Deil 1994

forme normale

forme relictuelle à Silene rosulata $x$ andryalifolia

Groupement à Sedum brevifolium (Transition vers le Tuberarion!)

Selaginello-Anogrammetum leptophyllae R. Molinier 1937 asplenietosum hemionitis Deil 1994

ADIANTETEA CAPILLI-VENERISBr.-B1. 1947

Adiantetalia capilli-veneris Br.-B1. 1931

Adiantion capilli-veneris Br.-Bl. 1931

Adianto-Hypericetum naudiniani Deil 1996

Adianto-Hypericetum pubescentis Varo Alcala \& Fernández Casas 1970

hypericetosum pubescentis cratoneuretosum filicini Deil 1996

Trachelio caerulei-Adiantetum $\mathrm{O}$. Bolós 1957

trachelietosum

phase optimale

phase terminale à Didymodon tophaceus

hypericetosum metroi Deil 1996

Eucladio verticillati-Adiantetum $\mathrm{Br}$--B1.

ex Horvatic 1934

eucladietosum

pteridietosum (Brullo et al. 1989) Deil stat. nov. 1996

Groupement à Adiantum capillus-veneris et Osmunda regalis Deil 1996 
CRITHMO-LIMONIETEA Br.-Bl. 1947

Crithmo-Limonietalia Molinier 1934

Daucion halophili Rivas-Martínez et al. 1990

\section{Limonietum emarginati Asensi 1984 carpobrotetosum edulis Deil 1994 \\ Leontodonto tingitani-Reichardietum picroidis Deil 1994}

RUDERALI-SECALIETEA CEREALIS Br.B1. 1936

Geranio purpurei-Cardaminetalia hirsutae Brullo in Brullo \& Marceno 1985

Valantio-Galion muralis Brullo 1985

Groupement à Mercurialis ambigua et Theligonum cynocrambe Deil 1994

(Transition vers le Geranio-Anthriscion et le Parietario-Galion murale!)

Urtico-Scrophularietalia peregrinae Brullo 1985

Allion triquetri O. Bolós 1957

Groupement à Succowia balearica

(aussi espèces transgressives du Geranio-Anthriscion!)

\section{BIBLIOGRAPHIE}

ALONSO, F.L. y J.M. EGEA -1994-Líquenes calcícolas y terrícolas de algunas localidades costeras de Marruecos. Acta Bot. Malacitana 19: 51-61.

ANDRIEUX, J. -1971- La structure du Rif central. Not. et Mém. Géol. Maroc 235. Rabat.

AVELLA, F.J. \& L.M. GONZÁLEZ - 1984- Monk Seal (Monachus monachus): A survey along the Mediterranean coast of Morocco. In: Ronald, K. \& Duguy, R. (eds.): The monk seals, Proc. 2nd. Int. Conf. $=$ Ann. Soc. Sci. Nat. Charenté Maritime 1984: 60-78.

BARKMAN, J.J., J. MORAVEC, \& S. RANCHERT -1986-Code of phytosociological nomenclature. Vegetatio 67: 145-195.

BLANCA LÓPEZ, G. y M. CUETO ROMERO 1985- Contribución a la flora de Granada: Algunas ligulifloras nuevas o interesantes. Lagascalia 13: 193-203.
BOULOS, L. -1972- Révision systématique du Genre Sonchus L. s.l. I: Introduction et classification. Bot. Not. 125: 287-319.

BOULOS, L. -1973-Révision systématique du Genre Sonchus L. s.I. IV: Sous-genre 3. Bot. Not. 126: 155-196.

BRAUN-BLANQUET, J. -1964- Pflanzensoziologie. Vienne.

BRAUN-BLANQUET, J. \& R. MAIRE -1924Études sur la végétation et la flore marocaines. Mém. Soc. Sci. Nat. Maroc 8: 5-244.

CANTÓ, P., S. LAORGA y D. BELMONTE -1986Vegetación y catálogo florístico del Peñón de Ifach (Penyal d'Ifach) (Alicante, España). Opusc. Bot. Pharm. Complutensis 3: 3-86.

Carte Géologique du Rif 1/50000, Feuille Rouadi 1987 - Rabat.

COUVREUR-LARAICHI, F. -1972- Les précipitations dans quelques stations du littoral de la mer d'Alboran. Rev. Géogr. Maroc 21: 85103.

DAUMAS, P., P. QUÉZEL \& S. SANTA -1952Contribution à l'étude des groupements végétaux rupicoles de l'Oranie. Bull. Soc. Hist. Nat. Afr. Nord 43: 186-202.

DEIL, U. -1992-Vicariance, pseudovicariance et correspondance - reflexions sur quelques notions de taxonomie et de syntaxonomie et les possibilitées d'une approche symphylogénétique. Coll. Phytosoc. 18: 165-178.

DEIL, U. -1994a- Felsgesellschaften beiderseits der Straße von Gibraltar. Hoppea, Denkschr. Regensb. Bot. Ges. 55: 757-814.

DEIL, U. -1994b- Klassifikation mit supraspezifischen Taxa und symphylogenetische Ansätze in der Vegetationskunde. Phytocoenologia 24: 677-694.

DEIL, U. -1996- Zur Kenntnis der AdianteteaGesellschaften des Mittelmeerraumes und angrenzender Gebiete. Phytocoenologia 26: 481-536.

DEIL, U. \& A. GALÁN DE MERA -1997Contribution à la connaissance des groupements rupicoles calcaires du Maroc. Bull. Inst. Sci. (in press).

DÍAZ GONZÁLEZ, T. E. -1989- Biogeografía y sintaxonomía de comunidades rupícolas (ensayo preliminar para una revisión de la clase Asplenietea trichomanis en la Península Ibérica, Baleares y Canarias). - Multicop. ined. IX Jorn. de Fitosoc. Alcalá de Henares 1989. 
DÍEZ GARRETAS, B. -1977- Algunas comunidades rupicolas en el Litoral de Málaga y Granada. Acta. Bot. Malacitana 3: 141-144.

EMBERGER, L. \& R. MAIRE -1941-Catalogue des Plantes du Maroc. Vol. IV. Alger.

ESCUDERO, A. \& S. PAJARÓN - 1994- Numerical syntaxonomy of the Asplenietalia petrarchae in the Iberian Peninsula. J. Veg. Sci. 5: 205-214.

JAHANDIEZ, E. \& R. MAIRE -1931/1934Catalogue des Plantes du Maroc. Vol. I-III. Alger.

JEANMONOD, D. -1984- Révision de la section Siphonomorpha Otth du genre Silene L. (Caryophyllaceae) en Méditerranée occidentale. II: Le groupe du S. mollissima. Candollea 39: 195-259.

JEANMONOD, D. -1985-Révision de la section Siphonomorpha Otth. du genre Silene L. (Caryophyllaceae) en Méditerranée occidentale. V: Synthèse. Candollea 40: 35-56.

JEANMONOD, D. \& G. BOQUET -1981- Remarques sur la distribution de Silene mollissima (L.) Pers. et des espèces affines en Méditerranée occidentale. Candollea 36: 279-287.

LIDEN, M. -1986- Synopsis of Fumarioideae (Papaveraceae) with a monograph of the tribe Fumarieae. Opera Bot. 88: 5-133.

LOIDI, J. \& A. GALÁN-MERA - 1989- Datos sobre la vegetación rupícola de la comarca madrileña de Torrelaguna (España). Studia Botanica 7: 159-171.

MARTÍNEZ PARRAS, J. M. \& M. PEINADO LORCA -1990- Ensayo sobre la vegetación rupícola basófila de la clase Asplenietea trichomanis en la provincia corológica bética. Acta Bot. Malacitana 15: 193-202.

MAURER, G. -1968-Les Montagnes du Rif Central. Trav. Inst. Sci. Chérif., Ser. 2, 14. Rabat.

MOTA POVEDA, J.-F., F. GÓMEZ MERCADO \& F. VALLE TENDERO -1991-Rupiculous vegetation of the betic ranges (south Spain). Vegetatio 94: 101-113.

PÉREZ CARRO, J., T.E. DÍAZ-GONZÁLEZ, M.P. FERNÁNDEZ ARECES y E. SALVO -1989Contribución al estudio de las comunidades rupícolas de la Cheilanthetalia marantomadarensis y Androsacetalia vandelii en la Peninsula Ibérica. Acta Bot. Malacitana 14: 171-191.

PÉREZ LATORRE, A.V., B. CABEZUDO y J.M. NIETO -1995- Nota fitosociológica sobre Rupicapnos africana subsp. decipiens en el sur de España. Acta Bot. Malacitana 20: 310-311. PÉREZ LATORRE, A.V., A. GALÁN DE MERA, U. DEIL y B. CABEZUDO -1996- Fitogéografía y vegetación del sector Aljibico (Cádiz-Málaga, España). Acta Bot. Malacitana 21: 241 -267.

PERIS, J.B., A. ROMO \& G. STÜBING -1995Sideritis fontiqueriana - eine neue endemische Art aus dem Rif-Gebirge (Marokko). Feddes Repertorium 106: 1-5.

PONS, A. \& P. QUÉZEL -1955- Contribution à l'étude de la végétation de rochers maritimes du littoral de l'Algerie centrale et occidentale. Bull. Soc. Hist. Nat. Afr. Nord 46: 48-80.

QUÉZEL, P. -1952- Contribution à l'étude phytogéographique et phytosociologique du Grand Atlas calcaire. Mém. Soc. Hist. Nat. Afr. Nord 50: 1-57.

QUÉZEL, P. -1953- Les Campanulacées d'Afrique du Nord. Feddes Repert. 56: 1-65.

QUÉZEL, P. -1957-Peuplement végétal des hautes montagnes de l'Afrique du Nord. Encyclopédie biogéographique et écologique 10. Paris.

RIBI, M. -1992-Étude écologique de la région du Parc National d'Al-Hoceima. Mém. 3ème cycle. I.A.V. HASSAN II. Rabat.

RIVAS-MARTÍNEZ, S. -1981- Etages bioclimatiques, secteurs chorologiques et séries de végétation de l'Espagne méditerranéenne. Ecologia Medit. 8: 275-288.

RIVAS-MARTÍNEZ, S., A. ASENSI, J. MOLERO MESA y F. VALLE -1991- Endemismos vasculares de Andalucia. Rivasgodaya 6: 5-76.

SAUVAGE, CH. \& J. VINDT, -1952- Flore du Maroc I. Trav. Inst. Sci. Chérif., Sér. Bot. 1. Rabat.

SAUVAGE, Ch. -1961- Recherches géobotaniques sur les suberaies marocaines. Trav. Inst. Sci. Chérif., Sér. Bot. 21. Rabat. 
SCHUHWERK, F. -1990- Relikte und Endemiten in Pflanzengesellschaften Bayerns - eine vorläufige Übersicht. Ber. Bayer. Bot. Ges. 61: 303-323.

VALDÉS, B., S. TALAVERA y E.F. GALIANO, (eds.) -1987- Flora Vascular de Andalucía Occidental. Vol. I-III. Barcelona.

VOGT, R. -1991- Die Gattung Leucanthemum Mill. (Compositae - Anthemideae) auf der Iberischen Halbinsel. Ruizia 10.

VOGT, R. -1994- Rhodanthemum laouense Vogt (Compositae, Anthemideae), a new species from Morocco. Willdenowia 24: 91-96.
VOGT, R \& Ch. OBERPRIELER. Studies in Centaurea Sect. Melanoloma in Morocco. Lagascalia. (in press).

Aceptado para su publicación en Julio de 1997

Direction des auteurs. U. DEIL: Université de Freiburg, Dept. de Géobotanique, Institut de Biologie II, Schänzlestr.1, D-79104 Freiburg, Allemagne. M. HAMMOUMI: Institut Agronomique et Vétérinaire Hassan II, Dépt. Ecologie végétale, B.P. 6202, Rabat-Instituts, Maroc. 